$\mathrm{Lu}(\mathrm{CHEN})$, p. 1

\title{
Identification and characterization of the hypoxia response regulatory elements in the accessible genome
}

\author{
Xi Lu, Xingqi Chen
}

Affiliations

Department of Immunology, Genetics and Pathology, Uppsala University, 75108, Uppsala, Sweden

Corresponding author: xingqi.chen@igp.uu.se

\section{Abstract:}

Hypoxia is commonly observed in the solid tumor and contributes to the drug resistance in cancer therapy. Deciphering the epigenetic feature under the hypoxia condition in the solid tumor is critical for us to understand the tumorigenesis and design the precision therapy. Using the time series of ATAC-seq data under the hypoxia treatment from the epithelia cells, we identified the hypoxia response regulatory elements (HRREs) in the accessible genome. We found that these different HRREs have unique genomic features and are enriched with different transcriptional factors (TFs). Our study provides insights into the chromatin structure response to the hypoxia treatment and identifies useful genomic features for a better understanding of the hypoxia biology in the solid tumor.

Key Words: Hypoxia, Chromatin, regulatory elements. 
Lu(CHEN), p. 2

\section{Introduction:}

33 Hypoxia is commonly observed in the solid tumor ${ }^{1-4}$. The cancer cells in the solid tumor are

34 found to alter their behaviors and metabolism under the hypoxia microenvironment, and

35 further resist the cancer therapy ${ }^{5,6}$. In the molecular level, hypoxia induces the production of

36 the hypoxia-inducible factors (HIFs) and other transcriptional factors (TF), modulates the

37 chromatin structure, and further changes the gene expression in the cancer cells ${ }^{7-10}$. Thus, a

38 detailed characterization of the chromatin structure changes under the hypoxia could help us

39 to understand the tumorigenesis in the hypoxia microenvironment of the solid tumor and to

40 design a precision therapy.

41 ATAC-seq technology is a powerful tool to decipher the chromatin structure with high

42 sensitivity ${ }^{11}$. Previously, we demonstrated that the low level of oxygen could modulate the

43 chromatin accessibility in the mouse breast tumor cell line 4T1 with ATAC-seq technology,

44 and we observed that the openness of a large number of regulatory elements are dynamically

45 changed during the time series of hypoxia treatment. We concluded that the hypoxia

46 microenvironment alone could reshape the transcriptional factor (TF) motif openness in the

47 genome wide ${ }^{12}$. However, to better understand the gene regulation under the hypoxia

48 environment, a detailed characterization of the dynamic changes of the hypoxia response

49 gene regulatory element is needed. Here, we performed a systematic annotation of the

50 hypoxia response gene regulatory elements in the 4T1 cells, and identified three different

51 types of hypoxia response gene regulatory elements (HRREs). We also found that there are

52 different genomic features and TFs bindings on different types of HRREs. 
Lu(CHEN), p. 3

\section{Results:}

\section{Identification of the hypoxia response gene regulatory elements}

57 To characterize the hypoxia response gene regulatory elements, we included all the ATAC-

58 seq data from the $4 \mathrm{~T} 1$ cells under $1 \% \mathrm{O} 2$ for various time points $(0,6,12,24,36,48$, and 72

$59 \mathrm{~h})^{12}$. We used the $\mathrm{Oh}$ as the normoxia control for the time series comparison. When we

60 compared the ATAC-seq peaks from each time point of hypoxia treatment with normoxia

61 control, we identified 6,750 significant differential peaks $(|\log F C|>1$, FDR $<0.01)$ (Figure 1a,

62 See methods). The openness of these significant differential peaks is modulated with the

63 hypoxia condition; thus, these 6,750 peaks are termed hypoxia response gene regulatory

64 elements (HRREs). Among these HRREs, a large proportion of regions, 5,015/6,750 (74.3\%),

65 start to open under the hypoxia condition, and only a small part of regions, 1,735/6,750

$66(25.7 \%)$, start to close with the hypoxia treatment (Figure 1a).

67

68 Next, we investigated the dynamics of these HRREs across the time points (see Methods).

69 Interestingly, we found the openness of 4,722 HRREs follows some trend to change during

70 the hypoxia treatment. With the unbiased cluster methods ${ }^{13}$, we identified three different

71 types of changes among these 4,772 HRREs, and named them clusters 1-3 (Figure b-e). The

72 HRREs in cluster $1(n=2,229)$ came to open gradually under the hypoxia treatment

73 compared with the control and reached the highest accessibility at $72 \mathrm{~h}$. We observed that the

74 HRREs in cluster $2(n=1,358)$ closed gradually during the hypoxia treatment. We also found

75 that the openness of HRREs in cluster $3(n=1,135)$ fluctuated during the time series of

76 hypoxia, but had the highest accessibility after the 72h hypoxia treatment (Figure 1f, 1g).

Characterization of the hypoxia response gene regulatory elements

Different types of HRREs under the hypoxia condition indicated different parts of genome responses differently to the hypoxia microenvironment. To understand the detailed mechanism of how different HRREs react differently to the hypoxia, we explored the genomic features of different HRREs in a systematic manner. When we performed the genomic annotation with all the HRRE peaks, and three different HRREs separately (Figure 2a), we found that the genomic annotation of HRREs in clusters 1 and 3 were similar to all the merged accessible peaks (Figure 2a), but the genomic annotation of HRREs in cluster 2 was quite different from the others (Figure 2a). In cluster 2, around $25 \%$ of the HRREs are from the promoter-TSS regions. This observation is further confirmed from the distance annotation strategy on the different groups of HRREs (Figure 2b), where it is clearly shown that more than $25 \%$ of the HRREs in cluster 2 are located within $1 \mathrm{k}$ distance from the transcriptional starting site. At the same time, the proportion of intergenic regions or distance between $10 \mathrm{~K}-100 \mathrm{k}$ in cluster 2 is significantly less than the other clusters. Since the HRREs in the clusters start to close under the hypoxia treatment, our result indicates that a big proportion of promoter regions in cluster 2 react to the hypoxia treatment. It was reported that the histone modification, $\mathrm{H} 3 \mathrm{~K} 27 \mathrm{ac}$, is associated with a higher activation of transcription and is found at both the proximal and distal regions of the transcription start site ${ }^{14}$. Thus, we

96 assumed that the HRREs in cluster 2 are enriched with the H3K27ac histone modification.

97 When we used the H3K27ac ChIP-Seq data from the normaixa condition to calculate the 98 H3K27ac distribution in the HRREs from each cluster (See Methods) ${ }^{15}$, we found indeed 
99 that there is a significant enrichment of $\mathrm{H} 3 \mathrm{~K} 27 \mathrm{ac}$ in cluster 2 compared with clusters 1 and 3

100 (Figure 2c). The significant enrichment of H3K27ac in cluster 2 indicates that a big

101 proportion of transcriptional active sites in the normoxia become silent in the hypoxia

102 condition.

103 It was shown that the accessibility of genomic regulatory elements is regulated by the 104 transcription factors binding on the genome ${ }^{16,17}$. To understand what types of transcriptional

105 factors regulate the different clusters of HRREs, we performed a motif enrichment for each

106 cluster of HRREs and identified the unique transcriptional factors (TF) for each cluster

107 (Figure 2d). (See Methods). We found that there are 152 TFs enriched in cluster 1(Table 1),

10876 TFs enriched in cluster 2(Table 2), and 91 TFs enriched in cluster 3(Table 3) (See

109 Methods and Supplementary materials). Interestingly, there are 42 shared TFs enriched in

110 all the clusters (significant, $\mathrm{q}<0.05$ ) (Table 4), and the common TFs in three different clusters

111 demonstrated that there are common mechanism for three HRREs under the hypoxia

112 treatment. After excluding the common TFs, we obtained a list of unique TFs identified for

113 each cluster (See Table 1-3), and these specific TFs regulate the dynamic changes of

114 chromatin accessibility in each cluster under the hypoxia treatment. Even Clusters 1 and 3

115 have the tendency of opening under the $72 \mathrm{~h}$ hypoxia treatment, but the unique list for their

116 TFs is quite different. There are 52 unique TFs for cluster 1, and 6 TFs for cluster 3.

117 Surprisingly, the hypoxia induced protein factors, HIF1a and HIF2a, are only enriched in

118 cluster 1, not the others (Figure 2d). The enrichment of hypoxia factors in cluster 1 probably

119 explains why it is continuously open during the entire treatment time. Even cluster 3 has a

120 tendency to stay open after $72 \mathrm{~h}$; however, there is some fluctuation in openness during the

121 time series treatment, which is probably because of the lack of hypoxia factor regulation on

122 the HRREs in cluster 3. At the same time, it also indicates that the HRREs in cluster 3 are

123 regulated differently from cluster 1 (Figure 2d). In cluster 2, 15 unique TFs were identified.

124 The Motif bHLHE41, Nkx6.1, and CRE could be seen when regulating the chromatin

125 structure $^{18-20}$, and it indicates that the decrease in the chromatin accessibility in cluster 2 is

126 regulated by these 15 TFs under the hypoxia environment.

127 In summary, the unique genomic feature and TFs enrichment in the different clusters of

128 HRREs potentially reflect the different mechanisms in the reactions to the hypoxia

129 microenvironment.

130 Discussion:

131 It was clearly shown that the hypoxia condition affects the gene expression ${ }^{21}$. Numerous

132 studies have demonstrated that the hypoxia microenvironment in the solid tumor contributes

133 to cancer development and progression ${ }^{6,14,22,23}$. The chromatin structure plays an important

134 role in controlling gene expression ${ }^{24}$. Thus, being able to decipher the chromatin regulation

135 under the hypoxia is vital to understand the solid tumorigenesis and to design a potential

136 therapy strategy. Here, we performed the fine characterization of how the genomic regulatory

137 elements respond to the hypoxia across the time series hypoxia treatment in the mouse

138 epithelial cell line. We also identified three types of hypoxia response regulatory elements

139 (HRREs) in the genome, and found that these different types of HRREs with different 
140 genomic features are enriched with different TFs. Our study provides insights into the

141 chromatin structure response to the hypoxia treatment and identifies useful genomic features

142 for a better understanding of the hypoxia biology in the solid tumor.

\section{Acknowledgements}

145 This work is supported by the Swedish Research Council (VR-2016-06794, VR-2017-02074

146 to X.C), Beijer Foundation, Jeassons Foundation, Petrus och Augusta Hedlunds Stiftelse,

147 Göran Gustafsson's prize for younger researchers, Vleugel Foundation, and Uppsala

148 University.

149

150

\section{Author contributions}

152 X.L. and X.C. designed the study, and wrote the manuscript together. X.C. supervised the 153 study.

154

\section{Competing Financial Interests statement:}

156 The authors declare no competing financial interests. 
Lu(CHEN), p. 6

\section{Materials and Methods:}

\section{Data availability:}

160 All the data used in this study were downloaded from the Gene Expression Omnibus under

161 accession number GSE112091.

\section{Data processing:}

164 ATAC-seq raw data is aligned to $\mathrm{mm} 9$, using Bowtie $2^{25}$ with the '-very-sensitive' parameter.

165 The properly paired reads with mapping quality larger than 30 were kept, and the duplication

166 reads were removed with Picard. Peak calling was performed with MACS2 using the

167 parameters $-\mathrm{q} 0.01$-nomodel -shift 0 , and the peaks overlapping with the genome black list

168 in the ENCODE were discarded.

Identification of the differential peaks and clusters:

171 Deseq $2^{26}$ was used to identify the differential peaks by comparing the ATAC-seq peaks from 172 each time point of hypoxia treatment (6h, 12h, 24h, 36h, 48h, and 72h) with ATAC-seq peaks 173 from the normal controls. The differential peaks list is identified with the following cu-toff

174 with: $p$-value $<0.01$, FDR $<0.01$, and the absolute value of $\log 2$ fold change $>1$. The 175 unbiased clusters from the differential peaks were tested with Mfuzz ${ }^{13}$. The Pearson

176 correlation of each cluster was used to verify the independence of each cluster. The heatmaps 177 of each cluster were generated using Deeptools ${ }^{27}$. Gene-element associations were then 178 filtered to a distance of $100 \mathrm{~kb}$ from element to TSS with HOMER ${ }^{28}$. KEGG and GO analyses 179 for each cluster were conducted with GREAT ${ }^{29}$.

\section{Genomic annotation and TF enrichment:}

182 The genomic annotation of each cluster was performed based on the genomic segmentation 183 of 3'UTR, 5'UTR, exon, intergenic, intron, non-coding, promoter-TSS, and transcription 184 terminal sites. The distance annotation of each cluster was chosen by using the transcription 185 start site of genes as the reference point, and the genome being separated into 5 parts: <1kbp, $1861 \sim 10 \mathrm{kbp}, 10 \sim 100 \mathrm{kbp}, 100 \sim 1000 \mathrm{kbp}$, and >1000kbp. The H3K27ac ChIP-seq data of 4T1 187 cells is downloaded from Gene Expression Omnibus ${ }^{15}$. The differential peaks from each 188 cluster were annotated with H3K27ac histone marker ${ }^{15}$. Transcription factors for each cluster 189 were determined using HOMER with a cut-off of q-value $<0.05$. The unique TFs for each 190 cluster are identified. 
Lu(CHEN), p. 7

192

193

194

195

196

197

198

199

200

201

202

203

204

205

206

207

208

209

210

211

212

213

214

215

216

217

218

219

220

221

222

223

224

225

226

227

228

229

230

231

232

233

234

235

236

237

238

239

240

241

\section{References:}

1 Li, X. F. \& O'Donoghue, J. A. Hypoxia in microscopic tumors. Cancer Lett 264, 172180, doi:10.1016/j.canlet.2008.02.037 (2008).

2 Walsh, J. C. et al. The clinical importance of assessing tumor hypoxia: relationship of tumor hypoxia to prognosis and therapeutic opportunities. Antioxid Redox Signal 21, 1516-1554, doi:10.1089/ars.2013.5378 (2014).

3 Harris, A. L. Hypoxia--a key regulatory factor in tumour growth. Nat Rev Cancer 2, 38-47, doi:10.1038/nrc704 (2002).

4 Gilkes, D. M., Semenza, G. L. \& Wirtz, D. Hypoxia and the extracellular matrix: drivers of tumour metastasis. Nat Rev Cancer 14, 430-439, doi:10.1038/nrc3726 (2014).

5 Brizel, D. M., Sibley, G. S., Prosnitz, L. R., Scher, R. L. \& Dewhirst, M. W. Tumor hypoxia adversely affects the prognosis of carcinoma of the head and neck. Int J Radiat Oncol Biol Phys 38, 285-289, doi:10.1016/s0360-3016(97)00101-6 (1997).

6 Muz, B., de la Puente, P., Azab, F. \& Azab, A. K. The role of hypoxia in cancer progression, angiogenesis, metastasis, and resistance to therapy. Hypoxia (Auckl) $\mathbf{3}$, 83-92, doi:10.2147/HP.S93413 (2015).

7 Koukourakis, M. I. et al. Hypoxia-inducible factor (HIF1A and HIF2A), angiogenesis, and chemoradiotherapy outcome of squamous cell head-and-neck cancer. Int J Radiat Oncol Biol Phys 53, 1192-1202, doi:10.1016/s0360-3016(02)02848-1 (2002).

8 Kumar, H. \& Choi, D. K. Hypoxia Inducible Factor Pathway and Physiological Adaptation: A Cell Survival Pathway? Mediators Inflamm 2015, 584758, doi:10.1155/2015/584758 (2015).

9 Choudhry, H. \& Harris, A. L. Advances in Hypoxia-Inducible Factor Biology. Cell Metab 27, 281-298, doi:10.1016/j.cmet.2017.10.005 (2018).

10 Ortiz-Barahona, A., Villar, D., Pescador, N., Amigo, J. \& del Peso, L. Genome-wide identification of hypoxia-inducible factor binding sites and target genes by a probabilistic model integrating transcription-profiling data and in silico binding site prediction. Nucleic Acids Res 38, 2332-2345, doi:10.1093/nar/gkp1205 (2010).

11 Buenrostro, J. D., Giresi, P. G., Zaba, L. C., Chang, H. Y. \& Greenleaf, W. J. Transposition of native chromatin for fast and sensitive epigenomic profiling of open chromatin, DNA-binding proteins and nucleosome position. Nat Methods 10, 12131218, doi:10.1038/nmeth.2688 (2013).

12 Chen, X. et al. Joint single-cell DNA accessibility and protein epitope profiling reveals environmental regulation of epigenomic heterogeneity. Nat Commun 9, 4590, doi:10.1038/s41467-018-07115-y (2018).

13 Futschik, M. E. \& Carlisle, B. Noise-robust soft clustering of gene expression timecourse data. J Bioinform Comput Biol 3, 965-988, doi:10.1142/s0219720005001375 (2005).

14 Carnero, A. \& Lleonart, M. The hypoxic microenvironment: A determinant of cancer stem cell evolution. Bioessays 38 Suppl 1, S65-74, doi:10.1002/bies.201670911 (2016).

15 Sonzogni, O. et al. Reporters to mark and eliminate basal or luminal epithelial cells in culture and in vivo. PLoS Biol 16, e2004049, doi:10.1371/journal.pbio.2004049 (2018).

16 Haberle, V. et al. Transcriptional cofactors display specificity for distinct types of core promoters. Nature 570, 122-126, doi:10.1038/s41586-019-1210-7 (2019).

17 Tsankov, A. M. et al. Transcription factor binding dynamics during human ES cell differentiation. Nature 518, 344-349, doi:10.1038/nature14233 (2015). 
bioRxiv preprint doi: https://doi.org/10.1101/2020.04.24.059105; this version posted April 25, 2020. The copyright holder for this preprint (which

was not certified by peer review) is the author/funder, who has granted bioRxiv a license to display the preprint in perpetuity. It is made available under aCC-BY-NC-ND 4.0 International license.

Lu(CHEN), p. 8

24218 Montagner, M. et al. SHARP1 suppresses breast cancer metastasis by promoting

243

244

245

246

247

248

249

250

251

252

253

254

255

256

257

258

259

260

261

262

263

264

265

266

267

268

269

270

271

272

273

274

275

276

277 degradation of hypoxia-inducible factors. Nature 487, 380-384, doi:10.1038/nature11207 (2012).

$19 \mathrm{Li}$, H. J. et al. NKX6.1 functions as a metastatic suppressor through epigenetic regulation of the epithelial-mesenchymal transition. Oncogene 35, 2266-2278, doi:10.1038/onc.2015.289 (2016).

20 Wen, A. Y., Sakamoto, K. M. \& Miller, L. S. The Role of the Transcription Factor CREB in Immune Function. J Immunol 185, 6413-6419,

doi:10.4049/jimmunol.1001829 (2010).

21 Chi, J. T. et al. Gene expression programs in response to hypoxia: Cell type specificity and prognostic significance in human cancers. Plos Med 3, 395-409, doi:ARTN e47

10.1371/journal.pmed.0030047 (2006).

22 Jing, X. et al. Role of hypoxia in cancer therapy by regulating the tumor

microenvironment. Mol Cancer 18, 157, doi:10.1186/s12943-019-1089-9 (2019).

23 Petrova, V., Annicchiarico-Petruzzelli, M., Melino, G. \& Amelio, I. The hypoxic tumour microenvironment. Oncogenesis 7, doi:ARTN 10

10.1038/s41389-017-0011-9 (2018).

24 Perino, M. \& Veenstra, G. J. Chromatin Control of Developmental Dynamics and Plasticity. Dev Cell 38, 610-620, doi:10.1016/j.devcel.2016.08.004 (2016).

25 Langmead, B. \& Salzberg, S. L. Fast gapped-read alignment with Bowtie 2. Nat Methods 9, 357-359, doi:10.1038/nmeth.1923 (2012).

26 Love, M. I., Huber, W. \& Anders, S. Moderated estimation of fold change and dispersion for RNA-seq data with DESeq2. Genome Biol 15, 550, doi:10.1186/s13059-014-0550-8 (2014).

27 Ramirez, F., Dundar, F., Diehl, S., Gruning, B. A. \& Manke, T. deepTools: a flexible platform for exploring deep-sequencing data. Nucleic Acids Res 42, W187-191, doi:10.1093/nar/gku365 (2014).

28 Heinz, S. et al. Simple combinations of lineage-determining transcription factors prime cis-regulatory elements required for macrophage and B cell identities. Mol Cell 38, 576-589, doi:10.1016/j.molcel.2010.05.004 (2010).

29 McLean, C. Y. et al. GREAT improves functional interpretation of cis-regulatory regions. Nat Biotechnol 28, 495-501, doi:10.1038/nbt.1630 (2010). 
Lu(CHEN), p. 9

279 Figures:
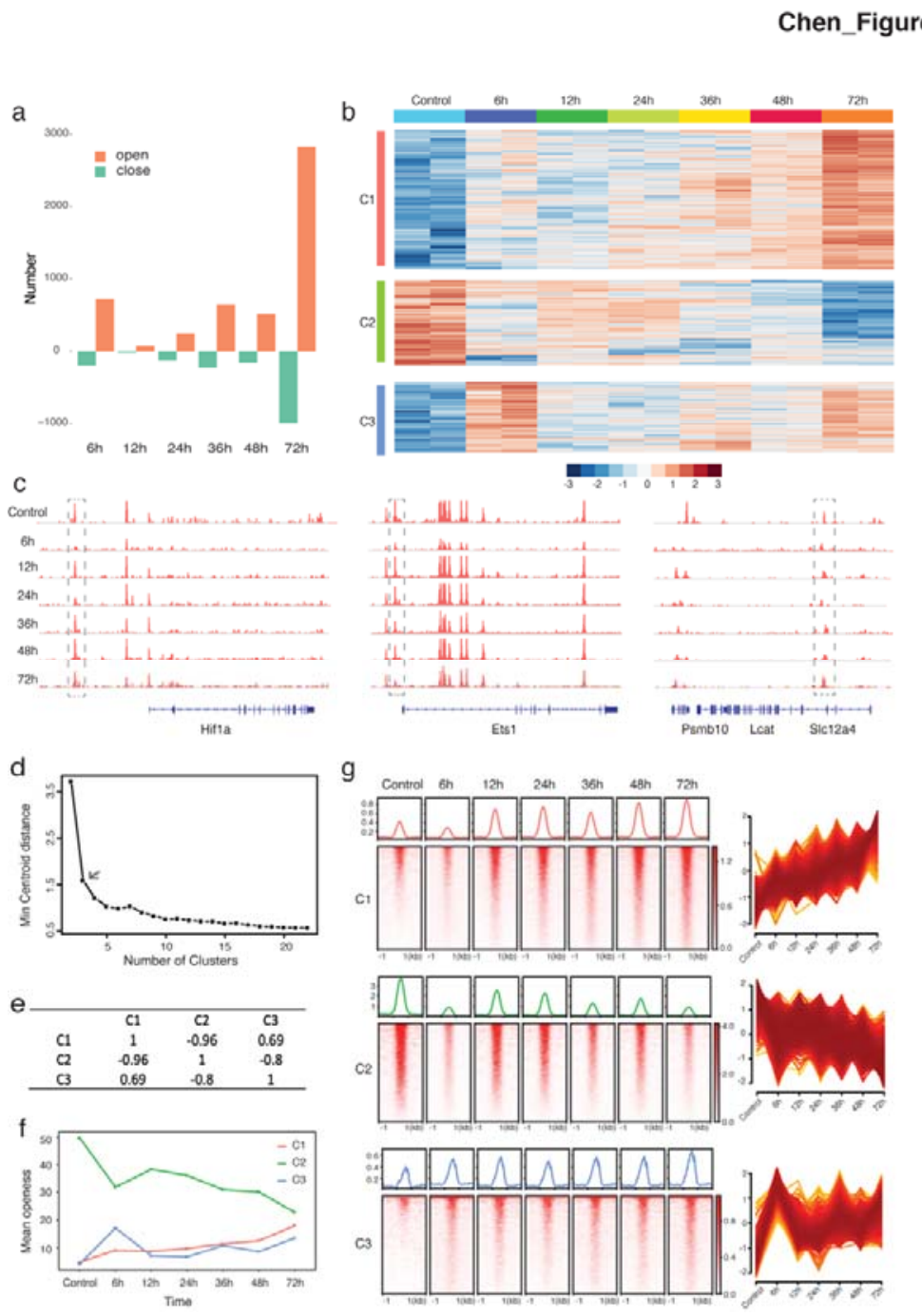

Figure 1. Identification of hypoxia response gene regulatory elements

a) Total accessible chromatin sites from different time points of hypoxia treatment were compared to the control (under normoxia condition). The more accessible chromatin sites are colored in orange, and the more close chromatin sites are colored in green.

b) The cluster of the hypoxia response gene regulatory elements (HRREs) following the time series of hypoxia treatment. $\mathrm{C} 1=$ cluster $1, \mathrm{C} 2=$ cluster 2 , and $\mathrm{C} 3=$ cluster 3 .

c) The representative genome browser track from the different cluster of HRREs. cluster1 (Hif1a), cluster 2 (Est1), and cluster 3 (Slc12a4). The grey box indicates the location of HRREs.

d) The centroid distance curve to determine the number of clusters for HRREs.

e) The pearson correlation among different HRREs clusters.

f) The average openness (calculated by RPKM) at each time point for each HRRE cluster.

g) Left: ATAC-seq signals for cluster 1 (C1), cluster 2 (C2), and cluster 3 (C3) at different time points. Right: the chromatin openness curve at different time points. 


\section{Figure 2. Characterization of hypoxia response gene regulatory elements}

a

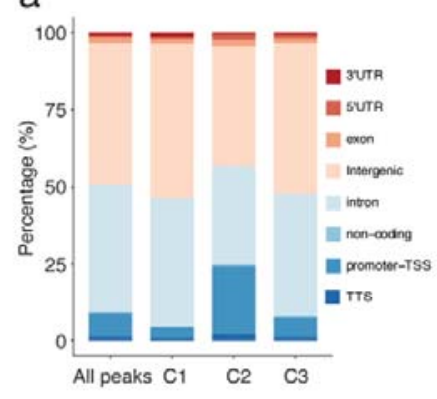

.

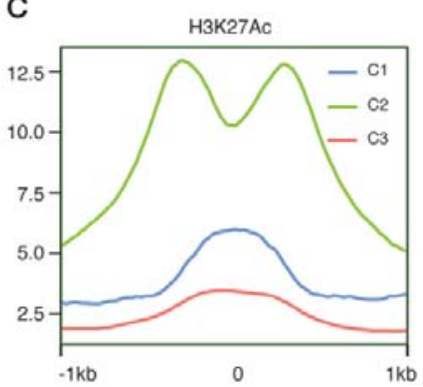

b

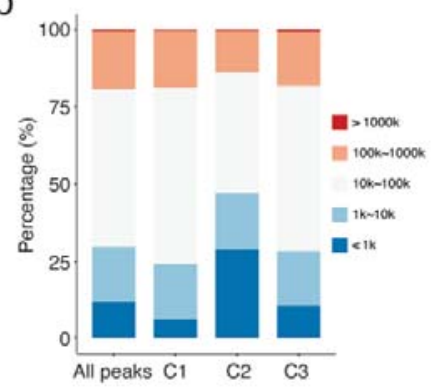

d

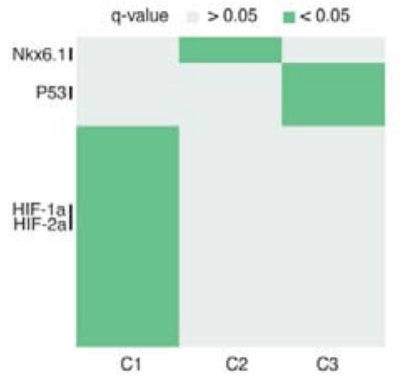

a) The genomic annotation of different hypoxia response gene regulatory elements (HRREs) clusters. $\mathrm{C} 1=$ cluster $1, \mathrm{C} 2=$ cluster $2, \mathrm{C} 3=$ cluster 3 , and $\mathrm{All}=$ all the accessible peaks from pool samples of all time points.

b) The annotation of the distance to transcription start sites (TSSs) from each HRRE clusters: cluster $1(\mathrm{C} 1)$, cluster $2(\mathrm{C} 2)$, and cluster $3(\mathrm{C} 3)$.

c) The enrichment of Histone marker H3k27ac for each HRRE cluster: 1 (C1), cluster 2 (C2), and cluster 3 (C3).

d) The heatmap of enriched transcriptional factor motifs on each cluster of HRREs. C1 $=$ cluster $1, \mathrm{C} 2=$ cluster 2 , and $\mathrm{C} 3=$ cluster 3 . 